\title{
Diverse therapeutic targets and biomarkers for Alzheimer's disease and related dementias: report on the Alzheimer's Drug Discovery Foundation 2012 International Conference on Alzheimer's Drug Discovery
}

\author{
Rachel F Lane*, Penny A Dacks, Diana W Shineman and Howard M Fillit
}

\begin{abstract}
The Alzheimer's Drug Discovery Foundation's 13th International Conference on Alzheimer's Drug

Discovery was held on 10-11 September 2012 in Jersey City, NJ, USA. This meeting report provides an overview of Alzheimer's Drug Discovery Foundation-funded programs, ranging from novel biomarkers to accelerate clinical development to drug-discovery programs with a focus on targets related to neuroprotection, mitochondrial function, apolipoprotein $\mathrm{E}$ and vascular biology.
\end{abstract}

\section{Introduction}

A multitude of factors lead to the onset of neurodegenerative diseases, including mitochondrial dysfunction, altered lipid metabolism and dysregulation of intracellular trafficking and signaling pathways. Ultimately these pathways, when dysfunctional, contribute to decreased synaptic activity in addition to the characteristic pathological hallmarks of Alzheimer's disease (AD), amyloid and tau accumulation. This conference highlights the diverse translational approaches scientists are undertaking to tackle this complex disease.

Plenary talks by Michael Gold (Allon Therapeutics) and Russell Swerdlow (University of Kansas) set the stage for the program with a focus on a systematic rather than target-based approach to AD intervention.

Swerdlow spoke on the bioenergetic changes with aging, such as altered glucose utilization, changes in

*Correspondence: RLane@alzdiscovery.org

Alzheimer's Drug Discovery Foundation, 57 W57th Street, Suite 904, New York, NY 10019, USA mitochondria morphology and size, and changes in the mitochondrial cyclooxygenase enzymes. The question is whether these changes are a cause or a consequence of the disease. Using cybrids (cells depleted of mitochondria and replaced with patient mitochondria from $\mathrm{AD}$ patients), Swerdlow and colleagues identified decreased mitochondrial function as a consequence of $\mathrm{AD}$. To increase mitochondrial mass and function in $\mathrm{AD}$, Swerdlow and colleagues provided evidence for PGC1 $\alpha$ as a novel target. Activation of PGC1 $\alpha$ with oxaloacetate boosts glycolysis and the mitochondrial mass supporting PGC1 $\alpha$ as a therapeutic target for AD and other neurodegenerative diseases.

Gold highlighted the development of Allon Therapeutics' neuroprotective peptide NAP (davunetide), a short eight-amino-acid fragment of the activity-dependent neurotrophic factor. NAP, thought to function as a microtubule stabilizer, effectively prevented tau hyperphosphorylation in mice. However, results released following the conference reported a failure to hit primary or secondary outcomes in a phase II/III trial in patients with progressive supranuclear palsy, an orphan indication that exhibits tau pathology similar to AD. The database of patient data, including magnetic resonance imaging and cerebral spinal fluid (CSF) measurements, will be made available to the research community and secondary analysis is underway.

\section{Amyloid beta and tau}

Metal-attenuating compounds have been the focus of a number of drug discovery programs in recent years based on the observations that removal of zinc and copper through clioquinol treatment decreases amyloid beta $(\mathrm{A} \beta)$ deposition in $\mathrm{AD}$ transgenic mice. Peter Kador (University of Nebraska Medical Center) presented data on the development of novel antioxidant, metal-chelating compounds. Kador described the novel system using 
light-induced retinal damage as a model of neurodegeneration, and the group is now performing prevention studies with their lead compound in an Alzheimer's transgenic mouse model.

Focusing on the downstream effects of $A \beta 42$ oligomer binding to neurons, Susan Catalano's (Cognition Therapeutics, Inc.) work has centered on the identification of a receptor for $\mathrm{A} \beta 42$ oligomer binding. While the receptor has not yet been disclosed, it is linked to increased risk for neurodegenerative diseases and clinical trials with drugs targeting this receptor have been undertaken for other indications. Cognition Therapeutics, Inc. has developed small-molecule drug candidates that disrupt the interaction between $A \beta 42$ oligomers and this receptor, rescue oligomer-induced vesicle trafficking defects, restore synapse numbers, and demonstrate some evidence for behavioral cognitive enhancement.

With a focus on tau pathology, Kun Ping Lu (Beth Israel Deaconess Medical Center, Harvard University) presented data supporting a novel target that prevents the formation of pathological tau conformations. Phosphorylated tau exists in two unique conformations, cis and trans, a transformation that is catalyzed by Pin1. Cisphosphorylated tau is the conformation of tau that preferentially accumulates into pathological tau species. $\mathrm{Lu}$ is therefore working to develop an immunotherapy approach specific for clearing pathological cis isoforms. A mAb has been developed and a proof-of-concept study in tau transgenic models is underway.

The primary dephosphorylating enzyme in the brain for tau and other proteins is protein phosphatase 2a (PP2A), a serine/threonine dephosphorylating enzyme. Jeffry Stock (Signum Biosciences, Inc.) presented the development of Cognion $^{\mathrm{Tm}}$ - a proprietary coffee extract enriched with eicosanoyl-5-hydroxytryptamide, an inhibitor of PP2A demethylation that decreases tau hyperphosphorylation. The group's studies have shown that eicosanoyl-5hydroxytryptamide is safe and reduces pathology and behavioral deficits in a mouse model of Lewy body disease and in a rat model of $\mathrm{AD}$ that has adenoassociated virusmediated increased expression of inhibitor $2 \mathrm{a}$, an inhibitor of PP2A. Signum Biosciences, Inc. has also partnered with Glaxo-Smith Kline to identify and develop next-generation PP2A demethylation inhibitors.

\section{Mitochondrial function}

Thiazolidinediones have been of interest in the diabetes and $\mathrm{AD}$ field for a number of years, but these drugs have been plagued with adverse cardiovascular events. In recent years, reports have indicated that the adverse effects of this class of compounds may be attributable to their peroxisome proliferator-activated receptor gamma activity while the beneficial insulin-sensitizing actions may be due to a direct effect on the mitochondria.
Jerry Colca (Metabolic Solutions Development Company) presented data to support development for a class of compounds that target the mitochondria but lack peroxisome proliferator-activated receptor gamma activity. In developing this lead series, Colca and colleagues identified a novel mitochondrial target of thiazolidinediones that regulate metabolism of pyruvate. Their lead compound, MSDC-0160, has shown positive effects in type 2 diabetes mellitus and a clinical trial is currently underway in early AD patients.

James Bennett Jr (Virginia Commonwealth University) is testing $R(+)$-pramipexole - an antioxidant that concentrates in the brain, specifically in the mitochondria - in early AD patients. A phase II study in patients with amyotrophic lateral sclerosis previously showed promising results, prompting this clinical trial in early $\mathrm{AD}$. While the investigators will include measurements of CSF isoprostanes as a marker of oxidative stress, they are additionally making CSF samples available to potential collaborators for analysis of other novel biomarkers.

\section{Synaptic function and cognitive enhancement}

Using phenotypic drug screens to identify compounds that target multiple age-associated pathologies, the laboratory of David Schubert has identified plant-based polyphenolics, including curcumin, as lead compounds for drug development. Marguerite Prior (The Salk Institute) presented work from Schubert's group on the development of curcumin derivatives with increased oral bioavailability and potency. The lead compound, J147, has demonstrated good medicinal chemistry properties, low toxicity, and evidence of neuroprotective, neurogenic, and cognitive enhancing properties in several animal models of $\mathrm{AD}$. The team is currently determining the mechanism of action of J147, which has been shown to modulate some of its effect through brain-derived neurotrophic factor.

Psychosocial stress can accelerate cognitive decline and pathology in AD and rodent models. John Csernansky (Northwestern University) presented evidence that this effect is due to corticotrophin-releasing factor rather than corticosterone. The team further demonstrated that the corticotrophin-releasing factor antagonists Antalarmin and R121919 can block the effects of corticotrophinreleasing factor on $A \beta$ levels in primary hippocampal cultures from mice. Corticotrophin-releasing factor antagonists, which are in various stages of discovery, may be available for repositioning to test for neuroprotective properties in stress-sensitive disorders.

Aged rodents with cognitive impairment are an alternative or complementary model for drug discovery on neuroprotective, synaptic plasticity, and cognitive enhancing targets. The development of allosteric ligands of neurotransmitter receptors offers the opportunity to 
modify endogenous neural activity while maintaining the temporal and focal components of endogenous signaling. Jerri Rook (Vanderbilt Center of Neuroscience Drug Discovery) presented evidence for VU0092273, a novel positive allosteric modulator of mGluR5 with improved physiochemical and pharmacokinetic properties. Rook and colleagues provided evidence that VU0092273 is effective at reversing age-related impairments in longterm potentiation and cognitive function.

Sharon Rosenzweig-Lipson (AgeneBio, Inc.) presented a different allosteric ligand approach based on evidence that positive allosteric modulators of GABA 5 receptors can improve memory in impaired aged rats. These ligands may be particularly effective during the early stages of $\mathrm{AD}$ in which hippocampal overactivity may impair cognitive function. Another program based on synaptic plasticity and cognitive function, presented by Mauro-Costa Mattioli (Baylor College of Medicine), showed enhancement of short-term to long-term potentiation using inhibitors of a novel kinase target, protein kinase $\mathrm{R}$.

\section{Apolipoprotein E}

While the $A P O E 4$ allele was identified as a significant risk allele for late-onset AD in the early 1990s, to date there are no late-stage development programs targeting this pathological mechanism. This is in part due to the unclear mechanism for contribution of the APOE4 allele to disease pathogenesis. Recognizing this gap in the field, in 2010 the Alzheimer's Drug Discovery Foundation initiated a call for proposals targeting apolipoprotein $\mathrm{E}$ (ApoE). This session highlighted a variety of approaches targeting ApoE mechanisms.

Programs presented in this session from Cheryl Wellington (University of British Columbia) and Rick Jack (Madera Biosciences, Inc.) focused on increasing levels of ApoE and the ApoE lipidating protein ABCA1 to stimulate $A \beta$ clearance from the brain. Jack presented the Madera development program for two lead compound series, MAD7012 and MAD7001. The MAD7012 series increased ABCA1 mRNA and secreted ApoE from human astrocytes. These effects are independent of the retinoid $\mathrm{X}$ receptor and liver $\mathrm{X}$ receptor, and thus will potentially avoid the negative side effects of hypertriglyceridemia and liver stenosis that are associated with activation of those receptors. MAD7012 is brain permeable following oral dosing. While the exact mechanism for action of this compound is unclear, the lead compound from this series is ready to enter in vivo proofof-concept studies.

In a screen to identify novel modulators of ApoE and $A B C A 1$, Wellington presented data on validation of initial hits from her screen of human astrocytes to increase ApoE. In secondary screens, progesterone and lynestrenol demonstrated dose-dependent activity, with lynestrenol showing the most robust effect on ABCA1 and $\mathrm{ApoE}$ via the liver $\mathrm{X}$ receptor. Wellington and colleagues are continuing screening of several libraries for additional modulators of ApoE and ABCA1.

Levels of the ApoE receptor LRP1 are decreased in the brain of AD patients. LRP1 is proposed to be one of the major ApoE receptors that mediate $A \beta$ clearance from the brain. Guojun Bu (Mayo Clinic) presented screens for small molecules that increase expression of $\mathrm{ApoE}$ and the ApoE receptors LDLR and LRP1. From these screens, Bu identified two compounds that increase ApoE secretion, ABCA1 mRNA levels and LDLR mRNA levels. Future studies will include counter-screening in APOE3/APOE4 astrocytes and optimization through a medicinal chemistry campaign. ApoE4 has also been hypothesized to exhibit a toxic gain of function and may function as a pathological chaperone promoting aggregation of $A \beta$.

Thomas Wisniewski (New York University) presented the development of novel competitive peptidomimetic inhibitors of the ApoE-A $\beta$ interaction using the $A \beta 12-$ 28 peptide with a proline substitution at position 18 . Preliminary data demonstrated that this peptide is effective at reducing amyloid burden and improving memory in several mouse models of $\mathrm{AD}$, including a mouse model of cerebral amyloid angiopathy (TgSweDI). Future development plans for this program include generation of shortened cyclic peptoids that are more resistant to proteolytic degradation with improved cell and blood-brain barrier permeability.

Robert Mahley (The J. David Gladstone Institutes) highlighted druggable pathways centered on the potential toxic gain of function of the ApoE4 protein, including increasing the mitochondrial protein cyclooxygenase-1 that appears to be decreased by proteolytic fragments of the ApoE4 protein. Through a screening campaign in N2A cells expressing ApoE4, Mahley and colleagues identified two classes of compounds that rescue cyclooxygenase- 1 levels. The group proposes to start mechanism of action studies for these molecules and to initiate a structure activity relationship campaign.

\section{Vascular targets}

Independent of the amyloid hypothesis, ApoE and ApoE receptors are closely correlated with risk for cardiovascular disease. The strong vascular component to $\mathrm{AD}$ has long been recognized, but the relative contribution to $\mathrm{AD}$ pathogenesis is unclear. Recent advances in the imaging of vascular occlusions during disease progression were presented by Nozomi Nishimura (Cornell University).

Using two-photon microscopy, Nishimura and the Schaffer laboratory discovered a $2 \%$ increase in small capillary occlusions resulting in stalled blood vessels in 
AD transgenic models. While some of these occlusions appear transient, at least one-half of them last for hours, resulting in reduced blood flow in downstream vessel branches. Further, they observed that these occlusions usually occur at the same position in the capillary, leading the team to identify leukocyte plugs (primarily monocytes) as the cause of the stall. Importantly, monocyte depletion significantly reduced the occurrence of stalls. While some targets that block leukocyte-endothelial interaction have not been effective at reducing stalls, the group continues to identify targets that reduce occlusions and restore blood flow.

\section{Biomarkers to accelerate clinical development}

Biomarkers that can effectively be used for early diagnosis or to track response to investigational therapies in clinical trials are critically needed for drug development. Marek Brzezinski (University of California, San Francisco) is seeking to identify those at high risk of postoperative cognitive decline, and is currently undertaking a clinical trial that will test whether preoperative brain $A \beta$ levels predict cognitive decline after surgery. The information from this work may accelerate clinical trials and identify high-risk patients in need of preventative treatment.

While AV-45 (Avid Radiopharmaceuticals), an amyloid imaging agent for $A \beta$, has recently been approved by the Food and Drug Administration, no imaging agent for tau is currently available. Jeff Kuret (Ohio State University) is developing tau imaging biomarkers for early diagnosis, differential diagnosis, and monitoring response to treatment. The major barriers for development are selectivity and the binding potential, and Kuret is working to overcome these barriers using pharmacokinetic modeling.

A second tau imaging program was presented by Victor Villemagne (Austin Health and The Mental Health Research Institute) with data for ${ }^{18} \mathrm{~F}-\mathrm{THK} 523$ in 20 patients. Interestingly, preliminary results showed higher white-matter retention in $\mathrm{AD}$ patients compared with control subjects, frontotemporal dementia patients, and progressive supranucleur palsy patients. Villemange reported that there appears to be a specific component to this largely nonspecific binding seen with other positron emission tomography ligands. However, at this time with small patient numbers - it is difficult to parse out specificity.

In the frontotemporal dementia field, Marcel Verbeek (Radboud University Medical Center, Nijmegen) presented data on optimizing and increasing the sensitivity of a TDP-43 ELISA for CSF measurement. The hypothesis is that TDP-43 and phospho-TDP-43 measures could improve the diagnosis of frontotemporal dementiatau versus frontotemporal dementia-TDP-43. While
TDP-43 can be measured in the CSF with this assay, the phosphorylated tau/tau ratio was still the most effective at differentiating patients. Combining the total tau measurement with total TDP-43 may further enhance the diagnostic utility, but requires further validation.

On a systems-wide approach, William Seeley (University of California, San Francisco) discussed large-scale brain network mapping techniques to understand selective neuronal variability. Such an approach could be more sensitive to early changes and thus be useful in monitoring disease progression. Further short-term reliability studies and longitudinal natural history studies are in development.

Ying Wu (Northshore University Health System Research Institute) presented data on the development of an automated toolbox that extracts regions of interest and improves the spatial resolution of magnetic resonance imaging scans. In addition to magnetic resonance imaging, fibers in the hippocampus and entorhinal cortex can be tracked. The techniques have been validated on normal patients and recruitment is underway to compare results for $\mathrm{AD}$ patients.

\section{Closing remarks}

In summary, the conference highlighted a portfolio of diverse therapeutic approaches to attack the multiple causes underlying AD. Alzheimer's is a complex disease, and diversifying the drug discovery pipeline beyond $A \beta$ targets is particularly important given recent clinical failures of several A $\beta$-targeted therapies. For more information on this conference and other Alzheimer's Drug Discovery Foundation scientific events, please visit http://www.alzdiscovery.org/index.php/news-events/ conferences.

\section{Abbreviations}

$A \beta$, amyloid beta; AD, Alzheimer's disease; ApoE, apolipoprotein E; CSF, cerebral spinal fluid; ELISA, enzyme-linked immunosorbent assay; mAb, monoclonal antibody; PP2A, protein phosphatase 2a.

\section{Competing Interests}

The authors declare that they have no competing interests.

\section{Acknowledgments}

This conference would not have been possible without the generous support of our sponsors: Pfizer Inc., Eli Lilly and Company, Elan Pharmaceuticals, Merck Research Laboratories, NeuroPhage Pharmaceuticals, JSW Life Sciences, Abbott Laboratories and Genentech, Satori Pharmaceuticals, Psychogenics, Janssen, GSK and Baxter.

Published: 4 February 2013

\section{doi:10.1186/alzrt159}

Cite this article as: Lane RF, et al:: Diverse therapeutic targets and biomarkers for Alzheimer's disease and related dementias: report on the Alzheimer's Drug Discovery Foundation 2012 International Conference on Alzheimer's Drug Discovery. Alzheimer's Research \& Therapy 2013, 5:5. 